\title{
Targeting with PRM
}

Parallel reaction monitoring (PRM)based targeted mass spectrometry is comparable in performance to selected reaction monitoring (SRM) but requires much less investment in assay development for targeted proteomics applications.

The term proteomics, to most, probably conjures up the notion of a discovery-based investigation. This is the practice of taking a sample, digesting the proteins into peptides, fractionating them with liquid chromatography, analyzing them by mass spectrometry and matching the spectra to databases to identify the peptides and hence the proteins from which they came. The field has grown by leaps and bounds in recent years, driven by advances in mass spectrometry instrumentation and supplemented by a plethora of methods ranging from sample processing to bioinformatics analysis.

Still, one is not always interested in performing an extensive discovery experiment. To address many types of biological, and certainly clinical, questions, researchers would like to closely follow what happens to a relatively small panel of proteins under different conditions, with high sensitivity and high quantitative accuracy. This is where the burgeoning field of targeted proteomics comes in.

The workhorse instrument of targeted proteomics is the triple quadrupole mass spectrometer, or QQQ. This instrument allows a technique called selected reaction monitoring (SRM) to be performed. SRM takes advantage of the ability of the QQQ to act as a mass filter such that assays can be developed to monitor a target analyte ion and one or more product ions generated by fragmentation in the mass spectrometer. These assays to detect the analyte ionproduct ion pairs are called SRM transitions, and they must be laboriously optimized to ensure the best performance. "You have to figure out what times your peptides elute, you have to figure out what transitions to look for, and then you have to write methods for your mass spectrometer that would include each transition, and each transition has to be optimized for each precursor, and it's just really a lot of work," explains Joshua Coon of the University of Wisconsin-Madison.
But with a technique described by Coon's lab and made possible by a new instrument, the Q Exactive (Thermo Scientific), the laborious development of SRM assays may be avoided. This instrument is much like a QQQ except that the third quadrupole is replaced with a high-resolution, high-mass accuracy Orbitrap mass analyzer. Whereas in SRM, all transitions are monitored one at a time, the Q Exactive allows parallel detection of all transitions in a single analysis. Coon and his colleagues thus dubbed the new technique 'parallel reaction monitoring', or PRM. Because all transitions can be monitored with PRM, one does not need to carry out laborious optimizations to generate idealized assays for selected transitions.

Still, it was not a given that the performance metrics of PRM would equal those of SRM. For one, the Orbitrap mass analyzer has a lower sensitivity and duty cycle than the QQQ. "We did the theoretical calculations before we did any experiments," Coon says. Once they got a hold of the Q Exactive instrument, they compared the performance of SRM and PRM by analyzing 25 heavy isotope-labeled synthetic peptides over a broad concentration range. But what the Orbitrap analyzer lacks in sensitivity "is made up for by the ability to look at all these products together," notes Coon. "The specificity of the mass accuracy allows you to weed out chemical noise." The performance of PRM was particularly good in complex backgrounds.

Part of the appeal of PRM, besides circumventing the need for extensive transition-assay optimization, is that the Q Exactive instrument is very flexible. "You have one instrument that can do [both] discovery and targeted work," Coon explains. This will allow researchers to use a discovery-based approach to identify a shortlist of interesting proteins and then use a targeted approach to follow those targets with high sensitivity under various conditions, all in a single experiment.

\section{Allison Doerr}

\section{RESEARCH PAPERS}

Peterson, A.C. et al. Parallel reaction monitoring for high resolution and high mass accuracy quantitative, targeted proteomics. Mol. Cell. Proteomics published online (3 August 2012). 